\title{
Practicing medicine on the high seas: a review of South African doctors' careers in cruise ship medicine
}

\author{
Carolyn Mary Lewis ${ }^{1}$, David Lee Skinner ${ }^{2}$, Roshen Maharaj ${ }^{3}$ \\ ${ }^{1}$ Division of Emergency Medicine, University of Witwatersrand, South Africa \\ ${ }^{2}$ Department of Anaesthesia and Critical Care, University of KwaZulu Natal, Nelson R Mandela School of Medicine, South Africa \\ ${ }^{3}$ Department of Emergency Medicine, Livingstone Hospital, Standford Road, Korsten, Port Elizabeth, South Africa
}

\begin{abstract}
Background: There has been an increase in the number of South African doctors working in the field of maritime medicine on board cruise ships. Despite this, there is a paucity of literature available addressing the epidemiology, level of expertise and continued medical education (CME) activities of cruise ship medicine. We aim to describe the demographics, qualifications and level of experience of South African doctors embarking on a career as a cruise ship doctor and assess ongoing CME and the future careers of doctors post cruise ship medicine.

Materials and methods: A survey was distributed to doctors either currently employed or previously employed as a cruise ship doctor from July 2012 to June 2017. The data obtained was used to describe the characteristics of South African doctors working in the field of cruise ship medicine.

Results: Of the 65 respondents, $61.5 \%$ were female. The majority of doctors were aged between 25 and 40 years. All South African doctors held a Bachelor of Medicine and Bachelor of Surgery degree, or equivalent, as a primary medical qualification and $46 \%$ held a Diploma in Primary Emergency Care prior to working at sea. The majority of doctors obtained certification in Basic Life Support, Advanced Cardiac Life Support, Paediatric Advanced Life Support and Advanced Trauma Life Support prior to joining cruise ship medicine. A wide range of courses and diplomas were undertaken by these doctors whilst working at sea. The majority of doctors opted for short to medium term contracts before returning to South Africa to pursue a land-based career, often by enrolling in postgraduate specialist training programmes.

Conclusions: Maritime medicine is an emerging field of emergency medicine in South Africa and requires a high standard of competence. A robust CME programme exists whilst working at sea. Most South African doctors return to South Africa to pursue a land based career, alleviating the potential concern that South Africa is permanently losing doctors to cruise ship medicine.
\end{abstract}

(Int Marit Health 2018; 69, 3: 171-175)

Key words: cruise ship medicine, maritime medicine, continuing medical education

\section{INTRODUCTION}

The global cruise industry has grown year on year and it is anticipated that 24 million passengers worldwide will have undertaken a cruise during 2016 [1].

The International Labour Organisation (ILO Convention 164 , article 8) specifies that all ships carrying 100 or more seafarers and ordinarily engaged on international voyages of more than 3 days' duration are required to carry a medical doctor on board. The qualification requirements of the medical doctor are not specified [2]; however, the American College of Emergency Physicians (ACEP) has published guidelines pertaining to the standard of care that should be provided within cruise ship medical facilities [3]. In terms of requirements pertaining to doctors, it is recommended that all clinical staff hold current full registration and a license to practice; have at least 3 years of post-graduate experience; have competence in emergency cardiovascular care and with minor surgical, orthopaedic and procedural skills 
such as suturing and fracture/dislocation management as well as procedural sedation skills. It is recommended that clinical staff be certified in advanced life support, such as Advanced Cardiac Life Support (ACLS) or Advanced Life Support (ALS) and at least one doctor on board should be certified in Paediatric Advanced Life Support (PALS) or Advanced Paediatric Life Support (APLS) and Advanced Trauma Life Support (ATLS).

Presently it is up to the individual cruise line companies to ensure that the doctors employed have the necessary qualifications, knowledge and experience to work at sea [3].

Personal correspondence with the medical directors of various cruise ship companies has revealed that there has been an increase in the number of South African doctors working in the field of maritime medicine on board cruise ships over the preceding 5 to 10 years.

Despite this increase in the number of South African doctors working in this field of maritime medicine, there is a paucity of local and international literature available addressing the epidemiology, level of expertise and continued medical education (CME) activities of cruise ship medicine; this is a very poorly studied area of medicine.

This study aims to describe the demographics, qualifications and level of experience of South African doctors embarking on a career as a cruise ship doctor. In addition, the CME activities available to cruise ship doctors and the future careers of doctors' post cruise ship medicine will be assessed.

\section{MATERIALS AND METHODS}

Our study analysed data obtained from a survey distributed to doctors, via Survey Monkey, either currently employed or previously employed as a cruise ship doctor from July 2012 to June 2017.

Application was made to medical departments of various cruise ship companies that employ South African doctors as part of their on board medical departments, as well as recruitment agencies recruiting South African doctors for cruise ship companies. Assistance was requested to distribute an email link to a Survey Monkey survey to all doctors employed over the preceding 5 years. The survey was both voluntary and anonymous.

The study population included all South African doctors employed on board cruise ships at any time from July 2012 until July 2017 inclusive. Doctors for whom no email address was available were excluded. Doctors with dual nationality but registered with the Health Professions Council of South Africa and/or residing in South Africa were included.

Data extracted included age, gender, primary qualification, additional qualifications and work experience prior to employment at sea. Data also included the length of time spent at sea (if resigned) or intended to be spent at sea, ongoing $\mathrm{CME}$ undertaken and careers pursued upon resigning from cruise ship medicine. Data was analysed using SPSS ${ }^{\circledR}$ (Statistical Packages for the Social Sciences) Statistics 24 (IBM Corp, USA).

Data security of the responses to the survey was maintained by access via a personal computer with password protection by the principle investigator, and once the survey was completed, data was stored offsite in a secure, password protected computer. Ethics approval was obtained from the University of KwaZulu-Natal Biomedical Research Ethics Committee (BE059/17) in South Africa.

\section{RESULTS}

For purposes of confidentiality a link to a Survey Monkey survey was distributed by participating cruise ship companies and recruitment agencies. One hundred and fifty surveys were distributed. Seventy-one doctors, of which 65 were South African, responded to the survey. Data from the survey results from South African doctors only were analysed.

The demographics were as follows: 40 (61.5\%) were female and 25 (38.5\%) were male. The majority of doctors were between 25 and 40 years old (Fig. 1).

Qualifications held prior to employment at sea are summarised in Table 1.

Figure 2 summarises the courses undertaken by doctors prior to employment at sea.

The majority of doctors, 54 (83\%), working at sea hailed from emergency medicine departments around South Africa, with the remainder from surgical, internal medicine and family medicine departments.

Qualifications obtained whilst working at sea are summarised in Table 2.

Figure 3 summarises the courses undertaken by doctors whilst at sea.

Eighty-eight per cent of doctors reported that their employer actively encouraged participation in continuing medical education.

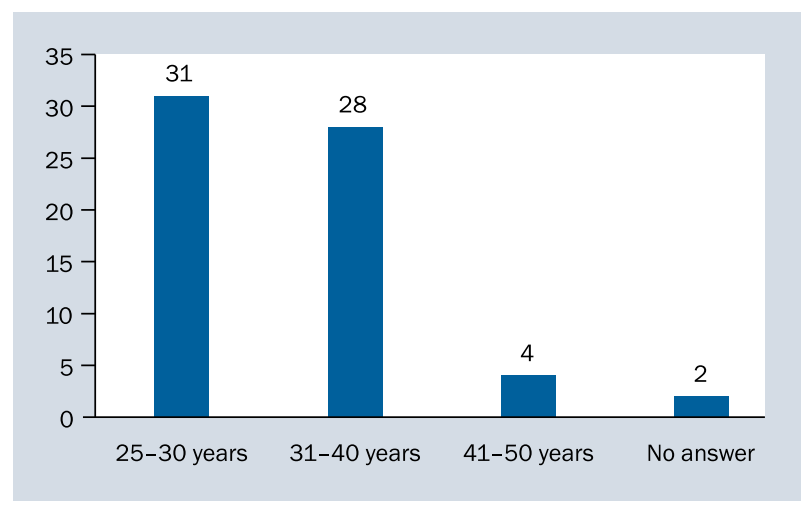

Figure 1. Age of doctors working in cruise ship medicine 
Table 1. Qualifications held prior to employment at sea

\begin{tabular}{llll}
\hline Qualification & & No. of doctors & Percentage \\
\hline Primary medical degrees & Bachelor of Medicine and Bachelor of Surgery & 65 & $100 \%$ \\
International degrees & & 0 & $0 \%$ \\
South African diplomas & Diploma in Primary Emergency Care (DipPEC) & 30 & $46 \%$ \\
& Diploma in HIV management (DipHIV) & 4 & $6 \%$ \\
& Diploma in Anaesthetics (DA) & 3 & $4 \%$ \\
& Diploma in Child Health (DCH) & 3 & $1 \%$ \\
& Diploma in Mental Health (DMH) & 1 & $1 \%$ \\
International diplomas & Diploma in Immediate Medical Care & 1 & $1 \%$ \\
& Diploma in Child Health & 1 & $1 \%$
\end{tabular}

Basic Assessment and Support in Intensive Care (BASIC) 1

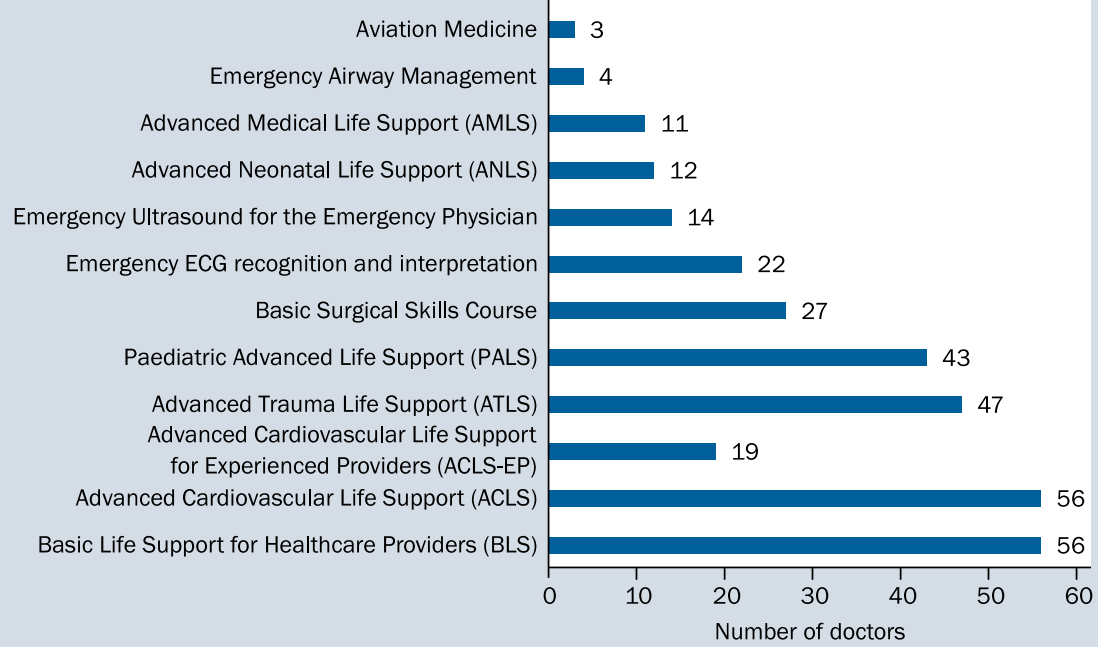

Figure 2. Courses undertaken by doctors prior to working in cruise ship medicine; ECG - electrocardiogram

Table 2. Qualifications obtained whilst employed at sea

\begin{tabular}{llll}
\hline Qualification & & No. of doctors & Percentage \\
\hline South African degrees & & 0 & $0 \%$ \\
International degrees & Masters in Aviation Medicine & 1 & $1 \%$ \\
& Masters in Paediatric Emergency Medicine & 1 & $1 \%$ \\
South African diplomas & Diploma in Family Medicine PG Dip (Fam Med) & 1 & $1 \%$ \\
International diplomas & Diploma in Immediate Medical Care & 1 & $1 \%$ \\
& Diploma in Remote and Offshore Medicine & 1 & $1 \%$ \\
& Diploma in Aeromedical Retrieval & $1 \%$ & $1 \%$
\end{tabular}




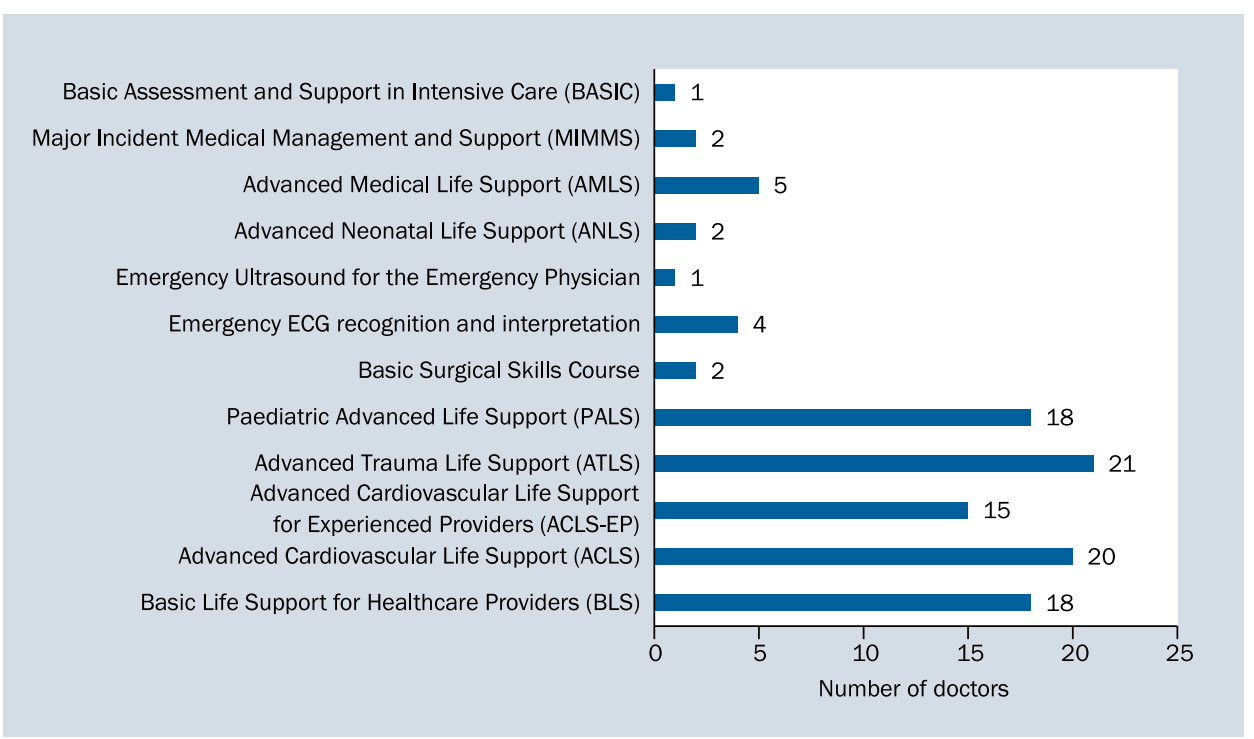

Figure 3. Courses undertaken by doctors whilst working in cruise ship medicine; ECG - electrocardiogram

Table 3. Careers pursued post cruise ship medicine

\begin{tabular}{lll}
\hline Current career pursuit & $\begin{array}{l}\text { No. of } \\
\text { doctors }\end{array}$ & Percentage \\
\hline Registrars (post graduate training post) & 7 & $27 \%$ \\
General practitioners & 3 & $11 \%$ \\
Medical officers in state practice & 2 & $8 \%$ \\
Medical officers in private practice & 1 & $4 \%$ \\
Specialist in state practice & 1 & $4 \%$ \\
Specialist in private practice & 2 & $7 \%$ \\
Remote and retrieval medicine & 1 & $4 \%$ \\
Occupational medicine & 1 & $4 \%$ \\
No longer in the medical field & 2 & $8 \%$ \\
Question unanswered & 6 & $23 \%$
\end{tabular}

Twenty-six (40\%) doctors had resigned from cruise ship medicine at the time of completion of the survey. Twenty-two of these doctors returned to South Africa with one doctor relocating to each of Australia, Madagascar, United States of America and the United Kingdom. Figure 4 summarises the duration of employment of doctors before resigning from cruise ship medicine.

Thirty-nine doctors (60\% of the total survey respondents) were still working at sea at the time of the survey and of these, $29(74 \%)$ indicated their intention to return to South Africa on resigning from cruise ship medicine. The length of time that these 39 doctors intend to remain as cruise ship doctors is summarised in Figure 5.

The future career pursuits of the 26 doctors who had resigned from cruise ship medicine at the time of survey completion are noted in Table 3.

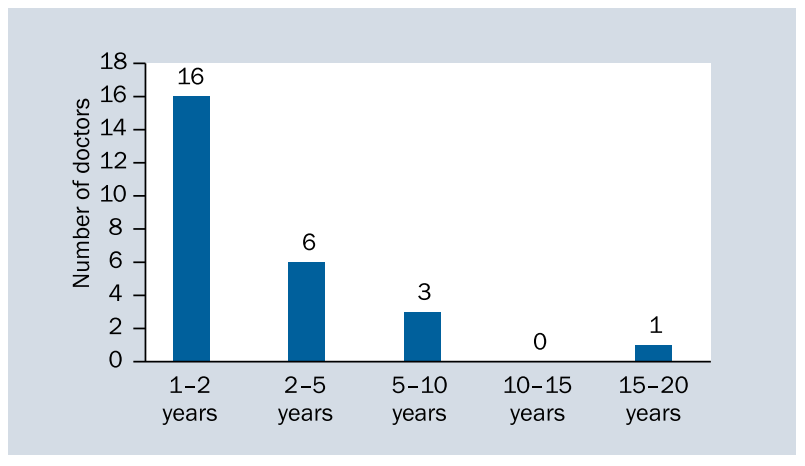

Figure 4. Duration of employment of doctors before resigning from cruise ship medicine

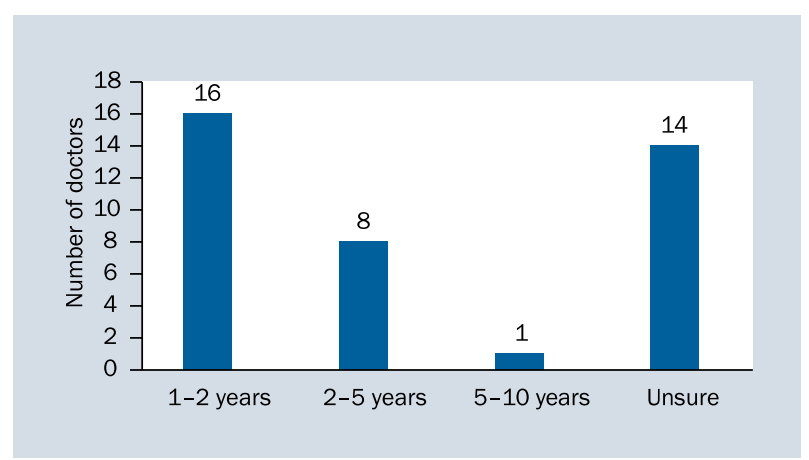

Figure 5. Intended length of time to be spent as cruise ship doctors

Of these 26 doctors, 7 (27\%) work in an emergency department with the remainder working in a variety of other disciplines. 
Sixty-five per cent of respondents noted that working at sea had influenced their future careers predominantly through development and fostering of an interest in the career that they are currently pursuing.

\section{DISCUSSION}

Cruise ship medicine has become an attractive option to South African doctors as it affords the opportunity to both travel and practice medicine in a remote and diverse environment whilst providing a competitive remunerative package. This has resulted in an increase in the number of South African doctors working in the field of maritime medicine on board cruise ships over the preceding 5 to 10 years.

In this study the majority of doctors working at sea are of a young age. Cruise ship medicine is contract based work which necessitates between 3 and 6 months (depending on the individual cruise ship company) at sea per contract. Thus it is anticipated that cruise ship medicine is likely to be a more attractive opportunity to younger doctors who have not yet established either a land based career or family and also seeking financial stability before pursuing post graduate studies.

The ACEP recognises cruise ship and maritime medicine as a section emergency medicine [3], and this affiliation between emergency medicine and cruise ship and maritime medicine is borne out in our survey of South African doctors. The majority of the survey participants had worked in an emergency department prior to working at sea. The Diploma in Primary Emergency Care in particular speaks to competence in emergency cardiovascular care and competence with minor surgical, orthopaedic and procedural skills such as suturing and fracture/dislocation management as well as procedural sedation skills as required by ACEP for doctors working at sea. Likewise the courses held by doctors prior to pursuing a career at sea is in keeping with ACEP requirements that clinical staff be certified in advanced life support with an additional recommendation for certification in either PALS or APLS and ATLS.

It would appear that there is a diverse range of academic opportunities available to doctors working at sea. Most doctors reported that their employer actively encouraged CME. This is reflected in the multitude of courses undertaken whilst working at sea. The majority of diplomas and degrees undertaken whilst working at sea are correspondence diplomas and degrees in keeping with opportunities available to doctors working in this remote and offshore site as well as the logistical challenges of this environment. Although remote and offshore, this does indicate that there are adequate electronic data communication portals at sea such that correspondence studies are both feasible and achievable.
There are a minority of doctors for whom cruise ship medicine appears to be a long-term career option with the majority opting for short to medium term contracts. Thereafter most doctors return to South Africa and pursue a land-based career often through specialising in their chosen field of interest. This would be in keeping with junior doctors who, once having travelled and obtaining financial stability, focus on furthering their careers through land-based studies and potentially also in establishing a family; both aspects that are difficult to accomplish whilst working in a remote and offshore environment. These results are in keeping with a local study outlining the career plans of final year medical students, thus alleviating the potential concern that South Africa is permanently losing doctors abroad [4].

\section{LIMITATIONS OF THE STUDY}

The study is limited by the agreed participation of the medical departments of cruise companies and recruitment agencies contacted by the principle investigator and by the accuracy of the databases of contact details for medical staff maintained by these companies and agencies. The study is further limited by doctors' responses to the emailed Survey Monkey survey.

\section{CONCLUSIONS}

Cruise ship and maritime medicine is an emerging field of medicine that currently attracts an increasing number of South African junior doctors. Our study highlights the high standard of competence required to work at sea and demonstrates a robust CME standard that strives to maintain these high levels of expertise whilst at sea. Most South African doctors return to South Africa to pursue a land based career.

\section{ACKNOWLEDGEMENTS}

The authors would like to acknowledge the assistance from medical departments of various cruise ship companies as well as two recruitment agencies within South Africa, Atlantic Medical Recruitment and Cruise Line Medical Recruitment, for their agreed participation in this study.

\section{REFERENCES}

1. Cruise Lines International Association. 2016. Cruise Industry Outlook. 2015. http://www.cruising.org/docs/default-source/ research/2016_clia_sotci.pdf (Accessed 10-Jan-2017).

2. Dahl E. Cruise ship doctor: demands and challenges versus qualifications and training. Int Marit Health. 2009; 60(1-2): 33-35.

3. American College of Emergency Physicians. PREP-Health Care Guidelines for Cruise Ship Medical Facilities (2014). https://acep. org/Physician-Resources/Clinical/PREP (Accessed 21-Jan-2017).

4. de Vries E, Irlam J, Couper I, et al. Career plans of final-year medical students in South Africa. S Afr Med J. 2010; 100(4): 227-228, indexed in Pubmed: 20459968. 